"C2020 IEEE. Personal use of this material is permitted. Permission from IEEE must be obtained for all other uses, in any current or future media, including reprinting/republishing this material for advertising or promotional purposes, creating new collective works, for resale or redistribution to servers or lists, or reuse of any copyrighted component of this work in other works." 


\section{A Review of Agency Architectures in Interactive Drama Systems}

\author{
Jonathan D. Moallem \\ Faculty of Engineering and Information Technology \\ University of Technology Sydney \\ Sydney, Australia \\ jonathan.moallem@student.uts.edu.au
}

\author{
William L. Raffe \\ Faculty of Engineering and Information Technology \\ University of Technology Sydney \\ Sydney, Australia \\ william.raffe@uts.edu.au
}

\begin{abstract}
This paper provides a review of the interactive drama field, attempting to create a taxonomy for classifying system affordances for emergence and authorial control, referred to in this paper as "agency architecture". The classification interactive drama systems according to a spectrum of agency architectures, helps to identify open questions in the field, providing a better understanding of which architectures would benefit from greater attention from the research community. In this paper, several key interactive drama systems from the field's literature are categorised by agency architecture. This is followed by a summary and analysis of their architectural classifications, alongside justifications for their assigned categories. It is then concluded with the identification of a number of research gaps, revealed by the compiled classifications, that highlight potential future research questions.
\end{abstract}

Index Terms-interactive drama system, interactive drama, agency architecture, review, emergence

\section{INTRODUCTION}

Over the past decades, many aspects of commercial video games have benefitted greatly from vast strides in technology, that have affected a multitude of aspects, including humancomputer interaction, player immersion, and infrastructure. However, while fidelity in graphics, audio, and even input has seen plenty of advancements, the fidelity of emotional and believable behaviour in non-player characters (NPCs) has remained fairly stagnant in being simplistic [1]. Imagine putting on your new virtual reality (VR) headset to see a beautifully realised world with high quality environment assets, realistic and immersive sound, as well as the ability to have your physical movements translated into that of your avatar's. The narrative of the game appears as compelling and realistic as real life. That is, until you begin to notice the familiar queues that railroad you in specific directions at certain points in time to encounter the same predictable circumstances of linear games past, and this suddenly shatters your emersion entirely. For this reason, it is necessary that the appropriate advancements in the field of artificial intelligence be made that developers can easily design believable characters and facilitate dynamic storytelling for video games so that the user is afforded the ability to suspend their disbelief [2].

The field of research surrounding this topic is most commonly referred to as interactive drama, narrative, or storytelling [3], and has been the topic of many an academic's study since the early 90s. In a 2009 review paper on the field, Arinbjarnar et al. defined the term "interactive drama" as:

[A game that takes] place within a virtual world in which the user has a high degree of freedom to physically and mentally interact with non-player characters and objects within a dramatically interesting experience which is different on every play and adapts to users interactions. [3]

A public interest for these kinds of games can be seen with the popularity of the field's better known projects such as Façade [1] and Galatea [4]. Both of them generating a great deal of buzz for the genre of interactive drama upon release due to their highly believable and dramatic scenes that adapted to player interaction.

There are three major challenges surrounding the issue of creating games with this depth of believable character: (1) the creation of an appropriate model for human behaviour, (2) the ability to orchestrate a narrative with characters utilising that model, and (3) the development of non-complex authorship tools. The creation of such a system does not just advance the artificial intelligence (AI) used for the styles of NPC that we see in current, popular games (such as RPGs), but also opens the door to social simulations for training and education [5], or entirely new genres, such as social physics games [6]. While these challenges have been met in a number of different ways academically, it is clear that the solutions provided thus far are not yet compelling enough to be adopted by industry in the mainstream games development community. This lack of adoption could stem from many of the potential criticisms of the field, including overcomplexity of models, the unwieldiness of dynamic narrative systems, or perhaps the authorship-intensive development process. One of the major differences between common architecture patterns used in the creation of interactive drama systems lies in its level of authorial control. Some systems will allow the naturally emergent features of the game to occur, creating a highly variable environment, whereas others implement a tightly controlled "director" system to oversee the game narrative's course. There is also a subset of these systems which provide their agents with autonomy, allowing for emergence, while also monitoring and adjusting their behaviour to retain greater 
authorial control over the game's ultimate narrative goals - a hybrid approach. These design considerations and features in a given interactive drama system that contribute to its level of authorial control during runtime will be referred to as its "agency architecture" throughout the course of this paper.

There are two major concerns that this paper addresses in its contribution to the field of interactive drama: firstly, in its ability to consolidate previous research in the field, and secondly, in categorising previous literature by their various agency architectures. While it was noted in Arinbjarnar et al.'s review paper that the field is rather disparate in nature [3], that is no less the case today with a great deal of alternative terminology being used, and siloed research conducted. This paper intends to perform broad research into major publications in the field of interactive drama, treating it as a historical account of its major contributions, which will provide a common account of the field's most influential works. Additionally, this paper aims to categorise these works by agency architecture, as so much of the definition of interactive drama is rooted in the degree of freedom, interactivity, and adaptability. Therefore it makes sense to provide a better overall understanding of how the concept of control is approached in each interactive drama system. As such, the classification of those works by their various agency architectures might provide a better understanding of which methods warrant greater attention and focus in future research.

The rest of this paper is organised in the following way: in Section [II it will provide an overview of the field, including its background, definitions for its common terminology, and a number of categories used in the classification of interactive drama systems. Following this, Section III. will discuss findings relating to key projects in the field's literature, and identify gaps in research for the field as well as potential open challenges.

\section{ABOUT INTERACTIVE DRAMA}

The field of interactive drama is a relatively large field in the realm of research, with literature on the topic spanning from games design, to computer science, social science, and even literary studies. This section aims to provide the reader with a basic understanding of the research field, firstly discussing it from a historical context, including its influences and most well known projects, and secondly by outlining two major concepts discussed in this paper: intelligent agents, and agency architecture.

\section{A. Generative Storytelling}

The field of interactive drama has been around since the early 90 s, but it was preceded by another field in computergenerated narrative, known as generative storytelling. The first major work in the field dates back to a thesis from 1976 titled "The Metanovel" that described the architecture and results for a program named TALE-SPIN [7]. The program was able to encode characters, their traits, and relationships to other characters in the system, and also deliver didactic tales including those characters in the style of Aesop's Fables.
Meehan notes that while the project was successful in creating a simulation for stories to unfold in, the system output was sometimes unnecessarily verbose, and characters often lacked context in basic matters, such as the difference between walking between two rooms, as opposed to up a flight of stairs.

Years later, a project following in the footsteps of TALESPIN was released under the name "UNIVERSE" [8]. The story generation system, unlike Meehan's work, focussed on the generation of believable plot outlines for soap operas, employing a plot-centric design. By authoring a library of "plot fragments" the system was able to connect complex, interwoven events between many characters (dynamically adding characters as required) in order to accomplish the narrative goals of the user. The plot outlines that the system could generate were rather convincing as works of television melodrama and achieved its goal in providing greater authorial control and delivering cohesive storylines. This being said, the system could have been vastly improved by fostering a better understanding of how far to generalise certain plot fragments [8], and lacked clarity in its character's motivations [3].

These two projects went on to inspire many of the key papers within with interactive drama field, including $\mathrm{Oz}$, Façade, and even Prom Week [2], [1], [6], [9].

\section{B. The Interactive Drama Genre}

There are a number of terms that refer to interactive drama (which was the original term for the genre), and the usage of those alternative terms often indicates the intention of the system's design, where for instance "interactive narrative" may be used when referring to projects showcasing high-level plot generation systems [3]. This paper uses the definition of interactive drama systems mentioned in the introduction of the work, highlighting three key elements. First and foremost to interactive dramas, they must be adaptable to player interaction as is the namesake of the genre, handling all user input gracefully to incorporate it into the narrative experience. Secondly, they must provide a wide possibility space so that successive play-throughs may include a non-trivial level of fundamental difference [3]. And thirdly, it is important for them to generate dramatically interesting experiences for the player. While it may be a highly subjective measure, it would defeat the purpose of interactive drama to not include any amount of dramatic interest at all.

Interactive dramas utilise a wide spectrum of features and technologies, however they often incorporate subsystems for procedural plot generation [10], [11], believable agents [6], [5], and natural language processing (NLP) [1], [12]. These subfields of interactive drama are seen by many as key research areas in the pursuit of creating better interactive drama systems. Two of the most well known projects in the field to date are $\mathrm{Oz}$ [2] and Façade [1].

Bates' paper on The Oz Project [2] detailed one of the first fully-featured interactive drama systems created, attempting to simulate autonomous characters that could exhibit emotion and general cognitive abilities. The game encompassed a small scene including four blob-like characters referred to as 
"Woggles", where three utilised a cognitive and emotional (AI) agent architecture, Tok [2], and one was controlled by the player [13]. The characters could communicate in relatively simple ways (such as distorting their body shape to greet each other), having the ability to engage in simple joint behaviours with one another, such as playing or showing aggression. He was also successful in being able to encode Woggles with distinct personalities that would be apparent in their behaviours.

Façade, created by Mateas \& Stern [1], imagined the player as the evening guest of a couple, who unwittingly entangles themself in the collapse of their host's marriage. As the night progresses, the (NPC) couple attempt to use the player's presence to take verbal jabs at each other following on into arguments, revelations and conversations over the future of the relationship, making for a short but high-intensity domestic drama [14]. The player could communicate in natural language, via typed text, and the characters could provide combinatory responses that incorporated dialogue, body language, and facial expressions. The project succeeded in generating buzz for interactive drama among the games industry for its ability to deliver believable character responses and control the flow of drama and tension, yielding a high level dramatic interest throughout the game.

\section{Agents in Interactive Drama}

In this paper, the term "agent" is used to refer to autonomous, decision making systems, which may act upon, and sense their environment, otherwise known as "intelligent agents" [15]. As such, this kind of AI is well-suited to the genre of interactive drama as it makes it easier to deal with a wide variety of player inputs, world states, and it often provides emergent gameplay and narrative. In this section, two key concepts in agent design, relevant to the analysis of interactive drama systems, are outlined: believable agents, and autonomous agents.

1) Believable Agents: There may be a perception that "believable agents" are the same as common game playing agents, however there is indeed a distinction between these two concepts. In a review of the field by Mateas [16], he suggests that it lies in the intention of the system - whether it is built in the context of evoking the perception of intelligence over servicing an objective measure. For instance, a game playing AI system will be built to solve objective problems like pathfinding or to simulate cognitive responses of the brain to a certain degree. Believable agents on the other hand are built to solve a subjective problem, which is how to make a character appear to exhibit intelligent behaviour that can often be flawed or suboptimal. The way we often describe what constitutes believable behaviour is personality, as a character is simply a simulacrum of life intending to masquerade as reality. Among the many considerations that go into building these systems, focusing on agent's ability to visualise expressions that hint at their internal states [17], and making sure that their actions appear to have causal connections [18] often go a long way to increasing their believability. There are a number of ways in which that has been achieved, such as with $\mathrm{The} \mathrm{Oz}$ Project's Woggles, which were given personality by varying the mappings between emotion and action [13] and Façade, in which characters had the use of gestures and expressions [1].

2) Autonomous Agents: Fully-autonomous agents are those that have freedom from external control. They govern their own state and actions while acting upon, or reacting to other entities, and, in purity, these AI systems should only be limited by the combinatorics of their own functionality on the world around them [1]. As entities that act on their own internal logics, they can be seen as simple systems, however their interactions with the game world and other entities lead them to exhibiting and contributing to emergent behaviours in complex systems. In practice, agent autonomy may only be the case to an extent, as many interactive drama systems allow for occasional non-autonomous control of autonomous agents, which will be referred to in this paper as semi-autonomous. For example, a game with semi-autonomous agents might have them act autonomously in an emergent environment until being interrupted by some kind of external system or inbuilt instruction to perform a specific action outside of their normal internal logics.

\section{Agency Architectures}

The interactive drama genre is primarily defined by providing players with a high degree of freedom and interactivity, as well as being able to adapt to a wide array of user interactions. Because of this, it is necessary to analyse the way in which interactive drama systems address the concept of control, which can be seen in their level of emergence. In a thesis on emergence in games, Sweetser defines emergent behaviour as:

... behaviour that occurs when simple, independent rules interact to give rise to behaviour that was not specifically programmed into a system. [19]

A typical example of emergence in commercial games can be seen with Far Cry 3 [20], in which tigers were scripted with the simple, independent rule of trying to kill other animals and humans. As a result, players often found that enemy outposts were attacked by tigers before the players had even arrived at them. While not having been specifically scripted into the experience, the design of the game had allowed for those events to occur through the natural emergence of the system. While this heavily lends itself to the facilitation of an emergent narrative, one of the the main goals of interactive drama is to create dramatically interesting experiences, which cannot be entirely guaranteed in a fully-emergent environment. With this being the case, interactive dramas require greater authorial control to be exercised by balancing freedoms and restrictions on the narrative throughout the experience.

In Jeff Gerke's book on authorial styles [21], "Plot Versus Character", he claims that there are two archetypes of writer: plot-first writers that focus on constructing a series of compelling events, and character-first writers that focus on creating believable characters. Likewise, this dichotomy appears in much the same way through interactive drama 
systems, as the authoring styles of each of the systems are constructed with a bias towards either a plot or character focus. This roughly translates in interactive drama system architecture to centralised (plot-driven) and and decentralised or emergent (character-driven), with each leading to a different basis for storytelling. However Sweetser's paper notes that while there are both emergent and scripted approaches to game design, the concepts are not mutually exclusive and thus a continuum exists between the two, where games may include emergent features, but also incorporate tightly scripted sequences or components [19]. This paper suggests that similarly to the aforementioned scripting-emergence continuum, interactive drama systems exist on a spectrum of "agency architecture" that ranges from centralised (scripted), to decentralised (emergent), as well as many hybrid approaches between. The rest of this section describes each architecture in greater detail, noting their strengths and weaknesses as well as mentioning exemplary works.

1) Centralised Systems: Centralised systems refer to interactive drama systems that utilise a "director", "drama manager", or "narrative manager". Complete control rests with the drama manager, which makes almost all decisions, and agents have little to no autonomy [22]. As an example, an agent in this system might be instructed to start a conversation with another character because the drama manager decided that it was required as part of the story. Centralised systems are often beneficial when the author requires a great deal of control over the narrative and can allow for story events to occur predictably. On the other hand, it reduces the amount of emergent possibilities that can occur, which reduces replayability and focuses gameplay on figuring out pre-authored puzzles [22]. Examples of centralised systems include Mimesis, with its "Mimesis Controller" system [12], and PaSSAGE, which adheres to a game event tree [11].

2) Decentralised Systems: Decentralised systems refer to interactive drama systems that simulate an emergent environment populated by fully-autonomous agents. In these systems, agents make their own decisions based on their own desires without regard to narrative objectives [22]. For example, an agent in this kind of system might decide to walk off to find food, because their internal logic dictated that they were hungry. Decentralised systems can be beneficial in cases where a large possibility space is desired, which increases replayability, and allows the player to solve puzzles by learning the mechanics of the underlying system [22]. One major drawback of this kind of system is that it lacks the inherent ability to control the narrative and can lead to unpredictable gameplay outcomes as a result. This style of system can be seen in both "FearNot!", which allowed all entities within scenes to act upon their own volitions [23], and Cavazza et al.'s fully autonomous simulation of the sitcom "Friends" [24].

3) Hybrid Systems: Hybrid systems refer to interactive drama systems that feature semi-autonomous agents, which may be adjusted or instructed on occasion by a drama manager. This is effectively a hybrid approach between centralised and decentralised systems and are built to varying degrees of authorial control. An agent in this system may decide to go find food because they are hungry, but the drama manager intervenes half way through to get them to speak with another character because they are required to do so as part of the story. This method attempts to mitigate the flaws of both centralised and decentralised systems by creating a hybrid between the two architectures, which in theory can provide both a robust possibility space, as well as mostly predictable events that can drive the narrative in a certain direction. While these systems attempt to strike a balance between the other two methods, it is important to keep in mind how that balance is made, and in what ways it is achieved. A hybrid architecture can be exemplified by Mirage, which allows the semi-autonomous agents to factor in goals set by the director system [17].

\section{DISCUSSION}

This review attempts to assess the field of interactive drama from the perspective of agency architecture, which is the degree to which the structure of a given system may provide affordance for, and balances, the concepts of emergence and control. This paper employs categorisation as the method to appropriately assess current literature in the field, where the systems listed in Table I can be grouped into codified architectures. The categorisation method divides the systems into three distinct architectural paradigms: centralised, decentralised, and hybrid. While centralised architecture refers to systems that strictly maintain narrative control through one, or many different, management-type systems, decentralised architecture refers to those which disperse control between the various entities in an emergent environment. Hybrid systems are simply systems that feature a combination of the two other methods. This section discusses the findings related to that categorisation process, providing justification for the classifications assigned to each of the interactive drama systems. Following this, a similar process is applied to a handful of commercial games to provide better context in regard to game system architecture. Finally, the findings from the compiled interactive drama system architectures from literature will be analysed in order to identify trends and potential research gaps.

\section{A. Agency Architecture in Literature}

After looking over the initial findings amongst the systems listed in Table I, it was clear that there was a necessity to increase the granularity with which this was done in order to gain relevant insights from the study. While the centralised and decentralised architectures could be easily identified, hybrid approaches were often difficult to place as they each exhibited varying levels of control and emergence. As such, agency architecture should rather be looked at as a spectrum as demonstrated in Fig. 1. All systems displayed in the figure would be classified as hybrid architectures as they incorporate both control and emergence through the incorporation of features like director systems and semi-autonomous agents. However, while Façade, for example, allows agents to respond based on their internal logics, they are only free to do so within the confines of fully-authored beats which they may be 
compelled to participate in at any time [1]. Therefore Façade would be far more centralised than Versu, which merely provides additional options to agents at given points in order to nudge them towards certain actions [22]. With this being the case, the hybrid category was replaced with two, more specific categories: Hybrid (Mostly Centralised), and Hybrid (Mostly Decentralised) where the difference between the two resides in whether the story-manager system had direct control over the actions of system agents.

Decentralised 2 Hybrid

Fig. 1. A visualisation of the agency architecture spectrum, featuring examples of Versu, Mirage, and Façade.

Table I indicates the categorisations applied to each system covered in this review. The first column contains the names of the systems, the second contains the year of the first paper they appear in, and the third contains their agency architecture. Explanations are provided in this section to justify the results of Table I in regard to their chosen architectures.

TABLE I

Interactive Drama Systems by Agency ARChitecture

\begin{tabular}{|c|c|c|}
\hline System & Year & Agency Architecture \\
\hline Oz & 1991 & Hybrid (Mostly Centralised) \\
\hline Erasmatron & 1999 & Hybrid (Mostly Centralised) \\
\hline Mimesis & 2001 & Centralised \\
\hline Friends Simulation & 2002 & Decentralised \\
\hline Façade & 2003 & Hybrid (Mostly Centralised) \\
\hline IDtension & 2003 & Centralised \\
\hline FearNot! & 2005 & Decentralised \\
\hline Thespian & 2005 & Decentralised \\
\hline IN-TALE & 2006 & Hybrid (Mostly Centralised) \\
\hline Mirage & 2007 & Hybrid (Mostly Centralised) \\
\hline PaSSAGE & 2007 & Centralised \\
\hline Prom Week & 2011 & Decentralised \\
\hline Versu & 2013 & Hybrid (Mostly Decentralised) \\
\hline Mysterious Murder & 2019 & Decentralised \\
\hline \multicolumn{2}{|c}{}
\end{tabular}

In the cases of Mimesis, which features the Mimesis Controller [12], and IDtension, which has a narrative sequencer [10], their inclusions of director-type systems to control agents and the game-world state plant them firmly in the centralised category. PaSSAGE performs user modelling in order to adapt the narrative for different types of players, doing this in a centralised manner, relying on a game tree to construct a branching narrative [11].

Systems like FearNot! and the Friends Simulation simply reside in the decentralised category as they both feature fullyautonomous agents in a simulated, emergent environment [23], [24]. Prom Week and Mysterious Murder on the other hand use a singular behaviour module for all agents, however agents still make decisions based on their own volitions, through a desire formation system [6] and Monte Carlo Tree Search [18] respectively, and thus should also be considered decentralised. Thespian, unlike many others in the decentralised category allows for authorial control though setting character goal weightings that influence their desire to execute narrative objectives [25]. This being said, it should still be considered decentralised as there is no runtime process that attempts to control agent behaviour.

The systems of Façade, IN-TALE, and Mirage, each have a director module that controls the behaviour of semiautonomous agents by setting their high-level goals, and allowing them to execute them autonomously in lower-level behaviours [14], [26], [17], thus they would belong among the more centralised hybrid systems. Oz and Erasmatron would also fall into this category, as they feature both autonomous agents, and drama manager systems that have the ability to modify the game-world state and agents directly to influence the course of the narrative [16], [3]. Finally, Versu solely occupies the category of more centralised hybrid systems, as it influences agent behaviour only by introducing additional actions that agents may decide to take part in at certain points in the narrative [22].

\section{B. Agency Architecture in Commercial Games}

While this paper primarily aims to assess the state of research in the field of interactive drama systems through academic literature, it is also useful to put those systems into perspective amongst commercial games. Few commercial games would fit exactly into the interactive drama genre as the genre is not often seen as commercially viable due to limited market appeal and the great deal of authoring effort required. However it should be noted that commercial games also exist on the agency architecture spectrum, as they too contain structures that afford either emergence or control over the player's actions and overall narrative.

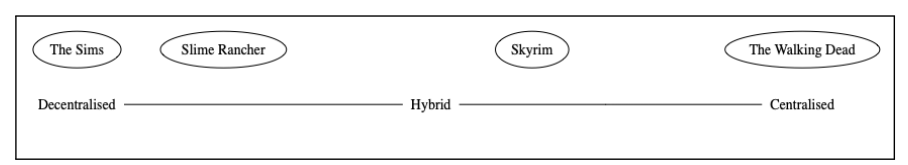

Fig. 2. A visualisation of the agency architecture spectrum, featuring examples from commercial games, including: The Sims, Slime Rancher, Skyrim, and The Walking Dead.

Fig. 2 plots a handful of commercial games against the agency architecture spectrum. Unlike the interactive drama systems from the literature, none of these games provide comprehensive documentation on their internal systems, and thus, the positions of each of the titles in the figure are only observed approximations. The systems displayed in the figure occupy classifications across the spectrum, including fullycentralised and fully-decentralised.

On the fully-centralised end of the spectrum is Telltale's The Walking Dead [27], which, much like a number of the interactive dramas discussed in the literature, utilises a game event tree that has the player follow a branching narrative. This method makes the game tightly scripted and does not allow for any emergent possibilities. On the other end of the spectrum is The Sims [28], which does not include scripted sequences, but 
rather allows for all entities in the system, known as "Sims", to interact in a fully emergent environment.

Like many modern, open-world type games, Skyrim [29] elects to provide the player with an emergent environment that allows the game's agents to react based on that environment. The game however sits towards the category of "mostlycentralised hybrid" as it also inhibits those emergent qualities with scripting for authored sequences, keeping entities at certain locations, managing quests, and much more. Finally, Slime Rancher [30] is a rare example of a commercial game that could potentially fall into the "mostly-decentralised hybrid" category as the game features a fully emergent environment, but also uses more subtle methods than direct scripting to control it. The game's designer, Nick Popovich, describes its emergent gameplay design as having deliberately placed agents in, and added mechanics to the game, which would lead to certain events occurring in the game naturally over time [31].

\section{Analysis \& Research Gaps}

Using the data compiled in Table 1 on the various agency architectures used by interactive drama systems, the field can be assessed as to the representation of various architectures in research. In this way, a number of research gaps can be identified for the purpose of better understanding the state and history of the field, as well as identifying areas that have been more broadly covered by past work.

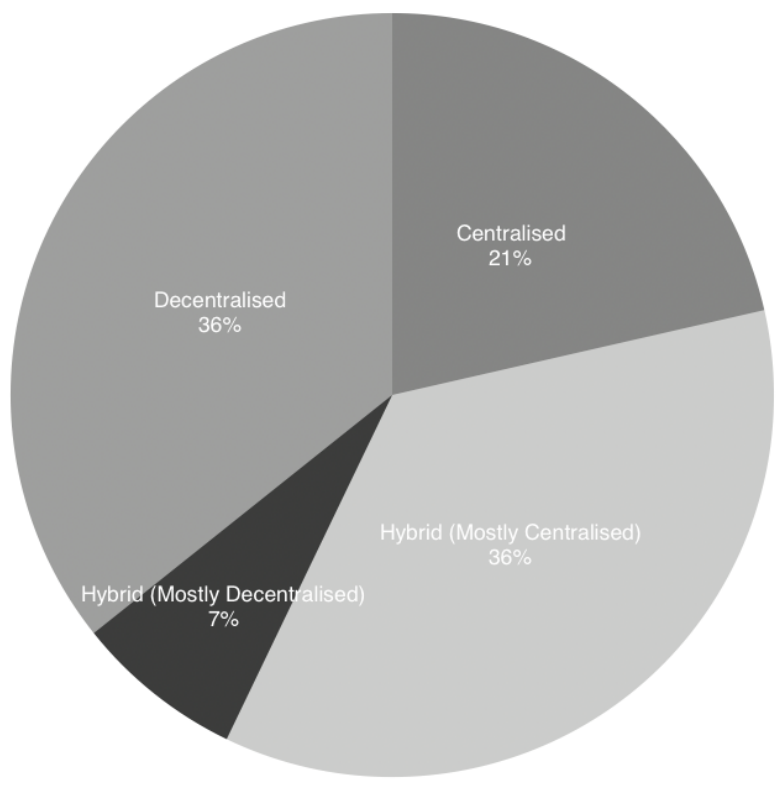

Fig. 3. A summary of agency architectures of systems listed in Table I

The results of the system's agency architecture categories in Table 1 are summarised by the pie chart in Fig. 3, with each slice representing the total number of items in each category. It can be seen that the categories of decentralised and more centralised hybrid systems account for the vast majority of the works covered in this paper, each representing 36\% (5 systems each). Centralised systems occupy $21 \%$ of all systems covered ( 3 systems), and only $7 \%$ are mostly decentralised hybrid systems (1 system). An observation that should be made, is that over time, there does not seem to be a particular trend towards any of the architectures, and as can be seen in Table [1] the presence of centralised, decentralised, and hybrid systems are fairly even over the timeline depicted. As mentioned previously, the two largest categories are decentralised, and mostly centralised hybrids. In the case of decentralised systems, most of the works discussed in this paper focus on believable agents over authorial control, and therefore make for less complex systems to build and manage. On the other hand, more centralised hybrid systems are the more heavy handed and straightforward of the two hybrid approaches to maintain authorial control over systems with large possibility spaces. These two approaches are well-covered by the research discussed in this review. Conversely, the smallest category, mostly decentralised hybrid systems, appears to have little representation among the works discussed in this paper. While the goal of the architecture is (similarly to the other hybrid category) to maintain narrative control within a highly emergent environment, this is made far more difficult by the director system's inability to directly control agents. As this category seems largely unexplored in the literature, this may present a research gap to be pursued in the interactive drama field. As previously described, a mostly-decentralised, hybrid system is one in which agents retain full autonomy throughout the course of the game, as the drama manager has no ability to directly instruct them or specify what their goals should be. The one interactive drama system described in this paper that implements this, Versu, achieves this by giving the drama manager the ability to modify the list of actions accessible to agents at certain points in the game. However there would be a number of other ways to go about this. A theoretical alternative to the Versu system might see the drama manager given the ability to affect world state, but not the agents. In this scenario, the author would create fully-autonomous characters for the scene, as well as script a drama manager that will attempt to push them towards story points naturally. For example, the drama manager might be given a narrative goal to have a character appear in the kitchen at $5 \mathrm{pm}$. To achieve this, the drama manager might cause a loud noise there at $4: 59 \mathrm{pm}$ in order to get the attention of the player. Alternatively, it could make the power in the house go out, forcing the character to walk through the kitchen on their way to find the fusebox. There would be two major benefits in crafting a system this way: introducing greater narrative control for fully emergent environments, and reducing the amount of effort in authoring content. By introducing a drama manager to an emergent environment, it will afford the designer greater authorial control in creating dramatically interesting content, and by preventing the drama manager from directly controlling the agents, their actions may appear more internally consistent while increasing player freedom. As mentioned, authoring effort would also be greatly reduced, requiring the narrative designer to simply create characters using personality traits or 
goals for the agents, and then create narrative goals for the drama manager to work towards.

\section{CONCLUSION}

Interactive drama systems allow developers to craft dramatically interesting digital experiences that adapt to users interactions, providing a great deal of freedom to the player. As a topic of research these systems have taken a number of forms and architectures, each with their own objectives and assumptions. A key differentiating factor between these systems is in their affordance towards authorial control and emergence, which is referred to in this paper as their agency architecture. This attribute has been codified into four distinct system categories, being centralised, decentralised, mostly centralised hybrid, and mostly decentralised hybrid. After summarising a number of works in the interactive drama field it was found that there were significantly fewer mostly decentralised hybrid systems, presenting a gap in the field's research, and an incentive to further explore the architecture. The exploration performed by this review paper of the interactive drama field has opened up a handful of questions for future research that may be useful in informing the direction of potential works in the field. Firstly, there is always the question of whether a broader study, with a larger sample size of interactive drama systems would affect the results, and potentially the conclusions drawn in this paper. Secondly, based on the research gap identified in Section III would there be value in pursuing further research into mostly decentralised hybrid systems? These questions may help to guide the field towards new understandings and paradigms for developing interactive dramas.

\section{REFERENCES}

[1] M. Mateas and A. Stern, "Façade: An experiment in building a fullyrealized interactive drama," in Game Developers Conference, vol. 2, pp. 4-8.

[2] J. Bates, "Virtual reality, art, and entertainment," vol. 1, no. 1, pp. 133 138.

[3] M. Arinbjarnar, H. Barber, and D. Kudenko, "A critical review of interactive drama systems," in AISB'09 Symposium: AI \& Games, Edinburgh, UK

[4] "Galatea, Versu, Character Engine/ Emily Short - Digital Cultures 2018." [Online]. Available: https://www.youtube.com/watch?v=S4hZOTRsILI

[5] S. C. Marsella, D. V. Pynadath, and S. J. Read, "PsychSim: Agent-based modeling of social interactions and influence," in Proceedings of the International Conference on Cognitive Modeling, vol. 36, pp. 243-248.

[6] J. McCoy, M. Treanor, B. Samuel, M. Mateas, and N. Wardrip-Fruin, "Prom Week: Social physics as gameplay," in Proceedings of the 6th International Conference on Foundations of Digital Games, pp. 319321.

[7] J. R. Meehan, "The metanovel: Writing stories by computer."

[8] M. Lebowitz, "Story-telling as planning and learning," vol. 14, no. 6, pp. $483-502$.

[9] J. McCoy, M. Treanor, B. Samuel, N. Wardrip-Fruin, and M. Mateas, "Comme il faut: A system for authoring playable social models," in Seventh Artificial Intelligence and Interactive Digital Entertainment Conference.

[10] N. Szilas, "IDtension: A narrative engine for Interactive Drama," in Proceedings of the Technologies for Interactive Digital Storytelling and Entertainment (TIDSE) Conference, vol. 3, no. 2, pp. 1-11.

[11] D. Thue, V. Bulitko, M. Spetch, and E. Wasylishen, "Interactive Storytelling: A Player Modelling Approach." in AAAI Conference on Artificial Intelligence and Interactive Digital Entertainment, pp. 43-48.
[12] R. M. Young et al., "An overview of the mimesis architecture: Integrating intelligent narrative control into an existing gaming environment," in Working Notes of the AAAI Spring Symposium on Artificial Intelligence and Interactive Entertainment. AAAI Press, pp. 77-81.

[13] "SFCI Archive:: The Oz Project: Woggles (1992)." [Online]. Available: https://vimeo.com/47883775

[14] M. Mateas and A. Stern, "Structuring Content in the Façade Interactive Drama Architecture." in AIIDE, pp. 93-98.

[15] M. Wooldridge, "Agent-based software engineering," vol. 144, no. 1, pp. 26-37.

[16] M. Mateas, "An Oz-centric review of interactive drama and believable agents," in Artificial Intelligence Today. Springer, pp. 297-328.

[17] M. S. El-Nasr, "Interaction, narrative, and drama: Creating an adaptive interactive narrative using performance arts theories," vol. 8, no. 2, pp. 209-240.

[18] C. Jaschek, T. Beckmann, J. A. Garcia, and W. L. Raffe, "Mysterious Murder - MCTS-driven Murder Mystery Generation," in 2019 IEEE Conference on Games (CoG), pp. 1-8.

[19] P. Sweetser, "An emergent approach to game design: Development and play."

[20] U. Montreal, "Far Cry 3," Ubisoft.

[21] J. Gerke, Plot Versus Character: A Balanced Approach to Writing Great Fiction. Penguin.

[22] R. Evans and E. Short, "Versu-a simulationist storytelling system," vol. 6, no. 2, pp. 113-130.

[23] R. S. Aylett, S. Louchart, J. Dias, A. Paiva, and M. Vala, "FearNot!-an experiment in emergent narrative," in International Workshop on Intelligent Virtual Agents. Springer, pp. 305-316.

[24] M. Cavazza, F. Charles, and S. J. Mead, "Character-based interactive storytelling," vol. 17, no. 4, pp. 17-24.

[25] M. Si, S. C. Marsella, and D. V. Pynadath, "Thespian: Using multiagent fitting to craft interactive drama," in Proceedings of the Fourth International Joint Conference on Autonomous Agents and Multiagent Systems, pp. 21-28.

[26] M. O. Riedl and A. Stern, "Believable agents and intelligent story adaptation for interactive storytelling," in International Conference on Technologies for Interactive Digital Storytelling and Entertainment. Springer, pp. 1-12.

[27] T. Games, "The Walking Dead," Telltale Games.

[28] Maxis, "The Sims," Electronic Arts.

[29] B. G. Studios, "The Elder Scrolls V: Skyrim," Bethesda Softworks.

[30] M. Park, "Slime Rancher," Monomi Park.

[31] "A Thousand Tiny Tales: Emergent Storytelling in Slime Rancher." [Online]. Available: https://www.youtube.com/watch?v=GbVFa89kUhw 